\title{
Primeiro registro de Chara indica e Chara zeylanica (Charophyceae, Charales, Characeae) em reservatórios do semiárido do estado do Rio Grande do Norte, Brasil
}

\author{
Gustavo Gonzaga Henry-Silva ${ }^{1 *}$ \\ Rafson Varela dos Santos ${ }^{1}$ \\ Rodrigo Sávio Teixeira de Moura ${ }^{1}$ \\ Norma Catarina Bueno ${ }^{2}$ \\ ${ }^{1}$ Universidade Federal Rural do Semi-Árido, Campus Mossoró \\ Avenida Francisco Mota, 572, CEP 59625-900, Mossoró - RN, Brasil \\ ${ }^{2}$ Universidade Estadual do Oeste do Paraná, Campus Cascavel \\ Rua Universitária, 2069, CEP 85819-110, Cascavel - PR, Brasil \\ * Autor para correspondência \\ gustavo@ufersa.edu.br
}

Submetido em 06/10/2012

Aceito para publicação em 29/04/2013

\section{Resumo}

No presente trabalho, apresentamos os primeiros registros das macroalgas Chara indica e Chara zeylanica para o estado do Rio Grande do Norte, Brasil, e para o semiárido nordestino. Exemplares de $C$. indica e $C$. zeylanica foram coletados nos reservatórios de Santa Cruz e Umari, respectivamente. Ambos os reservatórios estão localizados na bacia hidrográfica do Rio Apodi-Mossoró (oeste do estado), estando inseridos no bioma Caatinga.

Palavras-chave: Bioma Caatinga; Distribuição geográfica; Macroalgas; Ocorrência

\section{Abstract}

First record of Chara indica and Chara zeylanica (Charophyceae, Charales, Characeae) in the semiarid reservoirs the state of Rio Grande do Norte, Brazil. In the present study we present the first record of the macroalgaes Chara indica and Chara zeylanica for the state of Rio Grande do Norte, Brazil, and the semiarid northeastern. Specimens of C. indica and C. zeylanica were collected in Santa Cruz and Umari reservoirs, respectively. Both reservoirs are located in the river basin Apodi-Mossoró (Western State) in the Caatinga Biome.

Key words: Caatinga biome; Geographic distribution; Macroalgae; Occurrence 
A ordem Charales é formada por algas macroscópicas que se diferenciam pela organização do talo e pelas estruturas de reprodução sexuada (WOOD; IMAHORI, 1965). A família Characeae compreende seis gêneros (Chara, Lamprothamnium, Lychnothamnus, Nitellopsis, Nitella e Tolypella), podendo ser encontrada em todos os continentes, com exceção da Antártida. No Brasil esta família é representada pelos gêneros Chara e Nitella (BICUDO, 1979; VIEIRA JÚNIOR et al., 2003; PICELLI-VICENTIM et al., 2004; MCCOURT, 2011). As espécies do gênero Chara Linnaeus emend. C. Agardh, A. Braun são macroscópicas com hábito séssil, que se mantêm fixas no substrato por rizoides, apresentam râmulos verticilados diferenciados em nós e internós. Podem ser monoicas ou dioicas, com núcula em posição superior em relação ao glóbulo (PICELLIVICENTIM et al., 2004; MCCOURT, 2011; MEURER; BUENO, 2012).

Chara zeylanica Klein ex Willdenow, 1805 pode ser encontrada nos EUA, Brasil, Egito, Gana, Senegal, Paquistão, Sri Lanka, China, Taiwan, Tailândia, Austrália e Nova Zelândia (GUIRY; GUIRY, 2012). No Brasil, esta espécie já foi identificada nas regiões Norte (Pará), Centro-Oeste (Mato Grosso e Mato Grosso do Sul), Sudeste (Espírito Santo) e Sul (Rio Grande do Sul e Santa Catarina), mais especificamente nos domínios fitogeográficos da Amazônia, Mata Atlântica, Pampa e Pantanal. Chara indica C.G. Bertero ex C. Spengel, 1827 é encontrada no Caribe e no Brasil, sendo que em território brasileiro é relatada apenas no estado de Pernambuco no domínio fitogeográfico da Mata Atlântica (RODRIGUEZ, 1964; PRADO, 2003; ARAÚJO et al., 2010; ALVES et al., 2011; GUIRY; GUIRY, 2012). Em relação à morfologia, C. zeylanica é monoica caracterizada por possuir râmulos regularmente corticados, gametângios masculinos e femininos num mesmo nó, nó basal ocasionalmente fértil, curto, recoberto pela fileira superior de estipulódios. Chara indica é monoica caracterizada por possuir râmulos regularmente corticados, gametângios masculinos e femininos num mesmo nó, nó basal estéril e brácteas do nó basal muito menores que dos demais nós dos filídios.

Até o presente momento não há relatos na literatura da ocorrência de ambas as espécies em ambientes aquáticos do semiárido nordestino ou do bioma Caatinga e que informações sobre a distribuição e a ocorrência de espécies do gênero Chara no Brasil ainda são relativamente escassas, especialmente na região do semiárido. Neste contexto, o presente trabalho, que faz parte do levantamento de plantas aquáticas da bacia hidrográfica do Rio Apodi-Mossoró (HENRY-SILVA et al., 2010), tem como objetivo contribuir para o conhecimento da flora dos ecossistemas aquáticos do semiárido do Rio Grande do Norte.

Em agosto de 2011, foram realizadas coletas de amostras de água e de material vegetal nos reservatórios de Santa Cruz e Umari (RN), totalizando onze estações amostrais em cada um destes ambientes. Ambos os reservatórios estão localizados na bacia hidrográfica do rio Apodi/Mossoró na região do semiárido do Rio Grande do Norte (Figura 1). O reservatório de Santa Cruz está localizado no município de Apodi (0546’27’S; $\left.37^{\circ} 48^{\prime} 40^{\prime} \mathrm{W}\right)$ e foi concluído em 2002 por meio do barramento do rio Apodi/Mossoró, possuindo a segunda maior capacidade de reserva hídrica do Rio Grande do Norte. O reservatório de Umari está localizado no município de Upanema (0542'19”S; 37¹4’34”W) e foi concluído em 2002 por meio do barramento do rio do Carmo (Figuras 1 e 2).

O material vegetal foi obtido pelo processo de arranque manual em ambos os reservatórios. A análise dos exemplares foi feita com Microscópio Binocular Nikon E200 e Estereomicroscópio Binocular Physis. As exsicatas e o material fixado em formol $4 \%$ foram depositados no Herbário Dárdano de Andrade Lima da Universidade Federal Rural do Semi-Árido (Mossoró$\mathrm{RN}$ ), sendo que amostras foram também enviadas para o herbário da Universidade Estadual do Oeste do Paraná/ Cascavel-PR. A análise e a classificação do material vegetal (Characeae) seguiram as metodologias descritas por Bicudo (1974) e Krause (1997). Foram produzidos mapas de distribuição potencial no intuito de verificar a variação espacial do Índice de Estado Trófico (IET) de ambos os reservatórios, tendo por base os valores de fósforo, clorofila $a$ e transparência da água.

Exemplares de $C$. indica foram encontrados no reservatório de Santa Cruz (IET médio de 39), enquanto que $C$. zeylanica foi encontrada no reservatório de 
FIGURA 1: Fotografias do Reservatório de Umari - Upanema (A1: Zona fluvial; A2: Zona lacustre; A3: Barragem; A4; Bancos de Chara zeylanica). Fotografias do Reservatório de Santa Cruz - Apodi (B1: Zona lacustre; B2: Zona de transição; B3: Barragem; B4; Trecho com criação de tilápia do Nilo - Oreochromis niloticus).
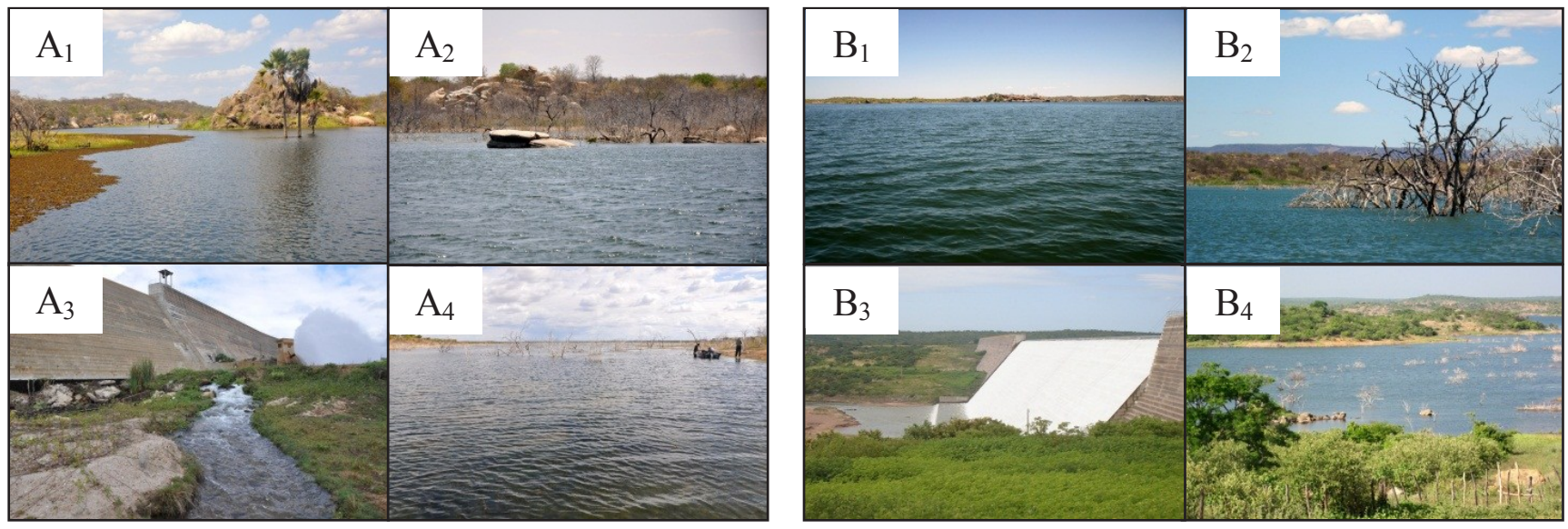

FIGURA 2: Reservatórios de Umari e Santa Cruz, localizados na Bacia Hidrográfica do Rio Apodi/Mossoró, semiárido do Rio Grande do Norte, Brasil. Os pontos nos mapas indicam os locais de ocorrência de C. zeylanica (Umari) e C. indica (Santa Cruz).

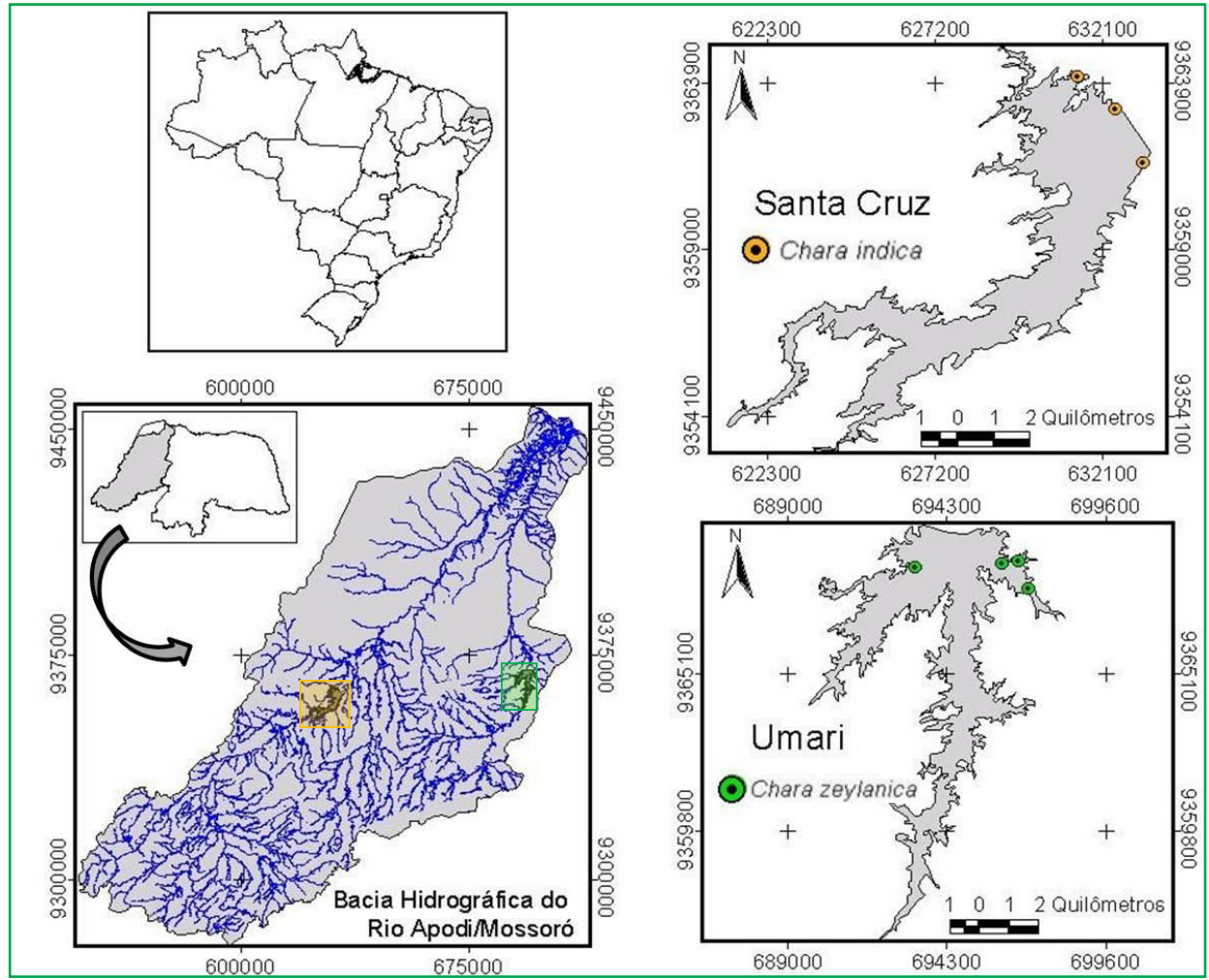

Umari (IET médio de 31). Ambos os reservatórios foram classificados como oligotróficos para o período de estudo. O mapa de distribuição potencial revelou que apenas a zona fluvial do reservatório de Santa Cruz apresentou características mesotróficas, no entanto neste trecho, não foram identificados exemplares de macroalgas.
Em ambos os reservatórios, bancos de $C$. indica e $C$. zeylanica foram encontrados apenas próximos à região litorânea da zona lacustre (Figuras 3 a 4).

As plantas do grupo Characeae tendem a desempenhar um papel ecológico importante na estruturação dos ambientes aquáticos, especialmente 
FIGURA 3: Índice de Estado Trófico (IET) para os reservatórios de Santa Cruz e Umari. Os pontos nos mapas referem-se aos locais de ocorência de C. indica e C. zeylanica em Santa Cruz e Umari, respectivamente.

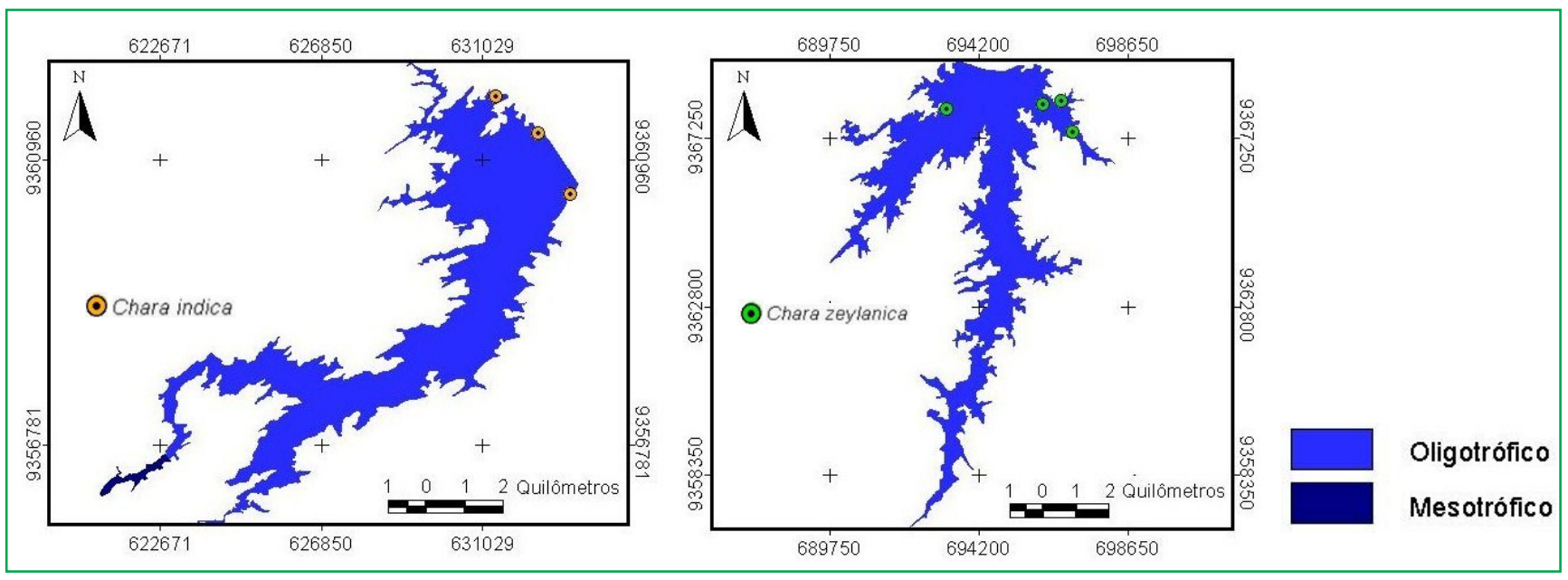

FIGURA 4: Bancos de C. zeylanica no reservatório de Umari (A) e C. indica no reservatório de Santa Cruz (B).
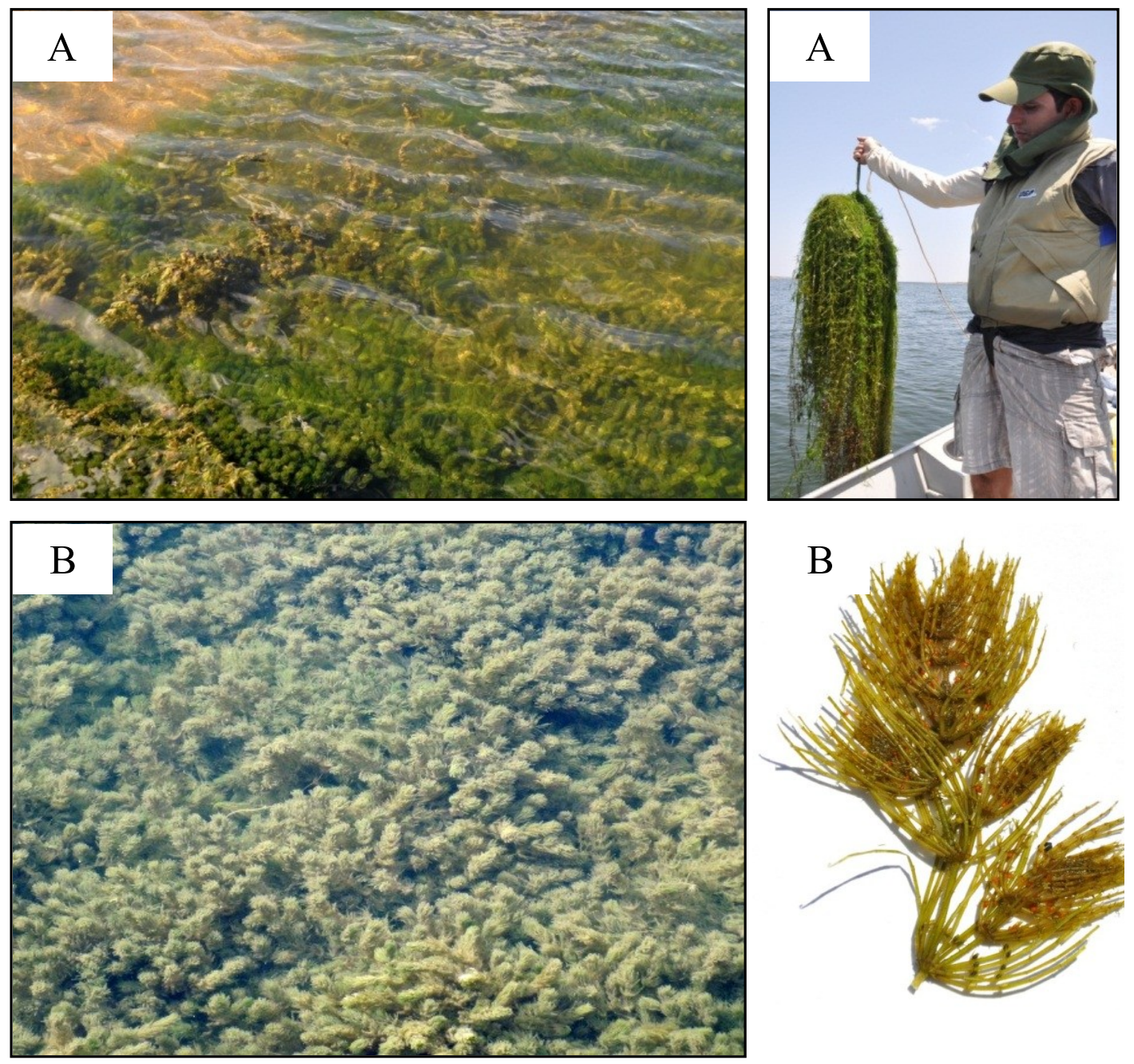
quando ocorrem em estandes com elevada biomassa, podendo atuar na absorção e ciclagem de nitrogênio devido principalmente as suas altas taxas de produção primária e os revestimentos de calcita que favorecem a retirada de nutrientes (COOPS, 2002; KUFEL; KUFEL, 2002). As plantas aquáticas submersas também podem contribuir para o aumento da diversidade alfa e na estruturação de habitats (CAMARGO et al., 2006; PELICICE et al., 2008). De fato, estudo realizado no reservatório de Lajeado (TO) constatou que áreas com a ocorrência de espécies de Chara apresentaram diversidade mais elevada de moluscos (AIRES, 2007). No reservatório de Santa Cruz foi observada a ocorrência do gastrópode Melanoides tuberculata em altas densidades associado à biomassa de $C$. indica. Este molusco de origem afroasiática consegue colonizar rapidamente os ambientes aquáticos alterados, limitando o crescimento ou atuando na exclusão competitiva de espécies nativas, especialmente daquelas pertencentes à família Planorbidae (FERNANDEZ et al., 2003).

Recentemente, alguns estudos realizados no Brasil têm ampliado a distribuição de algumas espécies do gênero Chara. Meurer e Bueno (2012) constataram a ocorrência de duas novas espécies (Chara hydropitys e C. rusbyana) para o reservatório de Itaipu e para o estado do Paraná (MEURER; BUENO, 2012). Bueno et al. (2009) documentaram $C$. hydropitys $[=C$. fibrosa var. hydropitys f. hydropitys] e C. guairensis pela primeira vez no Estado de Mato Grosso, enquanto que $C$. kenoyeri e C. socotrensis foram documentadas no Mato Grosso do Sul e Chara martiana em ambos os estados. Alves et al. (2011) registraram pela primeira vez $C$. guairensis e $C$. zeylanica para a Lagoa da Restinga do Massiambu (SC). Os registros de $C$. indica e C. zeylanica nos reservatórios de Santa Cruz e Umari ampliam a distribuição geográfica conhecida de ambas as espécies e do gênero Chara para o estado do Rio Grande do Norte e para o bioma Caatinga no semiárido nordestino. Futuros estudos de cunho ecológico poderão avaliar melhor a importância destas espécies para com estes ambientes aquáticos, além de demonstrar a sua importância para com a manutenção da biodiversidade local.

\section{Agradecimentos}

Ao técnico de laboratório Luiz Carlos Fernandes pelo auxílio nas coletas de campo. À Fundação de Amparo a Pesquisa do Estado do Rio Grande do Norte, pelo financiamento do projeto de pesquisa. A Coordenação de Aperfeiçoamento de Pessoal de Nível Superior (CAPES) e ao Conselho Nacional de Desenvolvimento Científico e Tecnológico (CNPq), pelas bolsas concedidas aos pesquisadores.

\section{Referências}

AIRES, S. E. P. S. Variação espacial e temporal da malacofalema associada a macroalgas aquáticas submersas na região litorânea do reservatório da UHE Lajeado, Porto Nacional - Tocantis - BRASIL. 2007. 29 f. Monografia (Bacharelado em Ciências Biológicas) - Universidade Federal do Tocantins, Porto Nacional. 2007.

ALVES, J. A.; TAVARES, A. S.; TREVISAN, R. Composição e distribuição de macrófitas aquáticas na lagoa da Restinga do Massiambu, Área de Proteção Ambiental Entorno Costeiro, Santa Catarina. Rodriguésia, Rio de Janeiro, v. 62, n. 4, p. 785-801, 2011.

ARAÚJO, A.; BUENO, N. C.; MEURER, T.; BICUDO, C. E. M. Charophyceae in Jardim Botânico do Rio de Janeiro. Lista de Espécies da Flora do Brasil. 2010. Disponível em <http:www. floradobrasil.jbrj.gov.br/2010/FB119551>. Acesso em: 01 dezembro 2010.

BICUDO, R. M. T. O gênero Chara (Charophyceae) no Brasil, 1: Subseção Willdenowia RD Wood. Rickia, São Paulo, v. 6, n. 1, p. 127-189, 1974.

BICUDO, R. M. T. O gênero Chara (Charophyceae) no Brasil, 1: Seção Charopsis (Kütz. Emend. Rupr., Leonh.) R. D. Wood. Rickia, São Paulo, v. 8, n. 1, p. 17-26, 1979.

BUENO, N. C.; BICUDO, C. E. M.; BIOLO, S.; MEURER, T. Levantamento florístico das Characeae (Chlorophyta) de Mato Grosso e Mato Grosso do Sul, Brasil: Chara. Revista Brasileira de Botânica, São Paulo, v. 32, n. 4, p. 735-750, 2009.

CAMARGO, A. F. M.; PEZZATO, M. M.; HENRY-SILVA, G. G.; ASSUMPÇÃO, A. M. Primary production of Utricularia foliosa L., Egeria densa Planchon and Cabomba furcata Schult \& Schult from rivers of the coastal plain of the State of São Paulo, Brazil. Hydrobiologia, Dorcrecht, v. 570, n. 1, p. 35-39, 2006.

COOPS, H. Ecology of charophytes: an introduction. Aquatic Botany, Amsterdam, v. 72, n. 4, p. 205-208, 2002.

FERNANDEZ, M. A.; THIENGO, S. C.; SIMONE, L. R. Distribution of the introduced freshwater snail Melanoides tuberculatus (Gatropoda: Thiaridae) in Brazil. The Nautilus, Sanibel, v. 117, n. 3, p. 78-83, 2003.

GUIRY, M. D.; GUIRY, G. M. Algae BASE. Galway: World-wide Electronic Publication, National University of Ireland. Disponível em: <http: www.algaebase.org>. Acesso em: 12 set. 2012. 
HENRY-SILVA, G. G.; MOURA, R. S. T.; DANTAS, L. L. O. Richness and distribution of aquatic macrophytes in Brazilian semi-arid aquatic ecosystems. Acta Limnologica Brasiliensia, Rio Claro, v. 22, n. 2, p. 147-156, 2010.

KRAUSE, W. Charales (Charophyceae). In: ETTL, H.; GÄRTNER, G.; HEYNING, H.; MOLENHAUSER, D. (Ed.). Subwasserflora von Mitteleuropa. Sttutgart: Gustav Fischer, 1997. p. 1-202.

KUFEL, L.; KUFEL, I. Chara beds acting as nutrient sinks in shallow lakes - a review. Aquatic Botany, Amsterdam, v. 72, n. 3, p. 249-260, 2002.

MCCOURT, R. M. Charales. 2011. Disponível em: <http:www. tolweb.org/Charales/20580/2011.11.22>. Acesso em: 22 nov. 2011.

MEURER, T.; BUENO, N. C. The genera Chara and Nitella (Chlorophyta, Characeae) in the subtropical Itaipu Reservoir, Brazil. Brazilian Journal of Botany, São Paulo, v. 35, n. 2, p. 219 232, 2012.

PICELLI-VICENTIM, M. M.; BICUDO, C. E. M.; BUENO, N. C. Flora ficológica do Estado de São Paulo: Charophyceae. São Carlos: Rima, 2004. 124 p.
PELICICE, F. M.; THOMAZ, S. M.; AGOSTINHO, A. A. Simple relationships to predict attributes of fish assemblages in patches of submerged macrophytes. Neotropical Ichthyology, Porto Alegre, v. 6, n. 4, p. 543-550, 2008.

PRADO, J. F. Characeae do Rio Grande do Sul, Brasil. 2003. 232 f. Tese (Doutorado em Botânica) - Universidade Federal do Rio Grande do Sul, Porto Alegre. 2003.

RODRIGUEZ, W. A. Novas Characeae na região Amazônica. Ciência e Cultura, São Paulo, 1964. v. 16, p. 154.

VIEIRA JÚNIOR, J.; NECCHI JÚNIOR, O.; BRANCO, C. C. Z.; BRANCO, L. H. Z. Characeae (Chlorophyta) em ecossistemas lóticos do Estado de São Paulo, Brasil: gênero Chara e distribuição ecológica. Hoehnea, São Paulo, v. 30, n.1, p. 53-70, 2003.

WOOD, R. D.; IMAHORI, K. A revision of the Characeae 1: monograph of the Characeae. Weinhen: J. Cramer, 1965. 904 p. 